\title{
Aplicación de vancomicina en polvo sobre el injerto. ¿Afecta la fusión de columna en conejos?
}

\section{Application of vancomycin powder on graft. Does the fusion of the rabbit's spine is affected?}

\author{
Guimbard-Pérez JH, ${ }^{*}$ Nicolás-Ortiz P, ${ }^{*}$ Cristiani-Winer M, ${ }^{*}$ Orosco-Falcone D, ${ }^{*}$ Gutiérrez N, * Pomba M* \\ Instituto Allende de Cirugía Reconstructiva.
}

RESUMEN. Introducción: Estudios in vitro sugieren que la vancomicina es altamente citotóxica para los osteoblastos. No obstante, diversos estudios clínicos in vivo no han aportado evidencias o determinado ese efecto en la osteogénesis. El objetivo del presente trabajo fue investigar el efecto de la vancomicina tópica, en un modelo de artrodesis lumbar sobre conejos blancos neozelandeses. Material y métodos: Estudio experimental randomizado doble ciego. Se realizó fusión espinal posterolateral a 30 conejos, divididos en dos grupos, A: injerto/placebo, B: injerto/vancomicina. Luego de sacrificarlos, se evaluó el callo óseo con tomografía axial, clasificándolos en tres grupos: sin fusión (0), fusión parcial/ incompleta (1) y fusión (2). Las muestras también fueron analizadas histológicamente. Las asociaciones entre presencia de fusión (completa/incompleta) y el grupo ensayado fueron estimadas con modelos log-lineales de Poisson con dos covariables. Las adherencias con las respuestas obtenidas histológicamente fueron estudiadas usando tablas de contingencia y test $\chi^{2}$. El nivel de significación se fijó igual a 0.05 . Resultados: El tratamiento con vancomicina tiene 30\% (0.30. IC 95\%: 0.12-0.94) menos probabilidad respecto al grupo placebo de presentar fusión completa. En otros términos, el grupo vancomicina tiene 2.3 veces (IC 95\%: 1.02-4.91) más probabilidad, respecto del placebo, de tener fusión incompleta. Conclusión: La aplicación de vancomicina en polvo mezclada con injerto reduce $30 \%$ las tasas de fusión, pero a dosis cinco veces más alta que las usadas rutinariamente en artrodesis por el equipo médico de los autores.

Palabras clave: Fusión, vancomicina, injerto, revisión.
ABSTRACT. Introduction: In vitro studies suggest that vancomycin is highly cytotoxic for osteoblasts. However, several clinical studies in vivo have not provided evidence or determined that effect on osteogenesis. The aim of this work was to investigate the effect of topical vancomycin on a lumbar arthrodesis model on New Zealand white rabbits. Material and methods: Doubleblind randomized experimental study. Posterolateral spinal fusion was performed on 30 rabbits, divided into two groups, A: graft/placebo, B: graft/vancomycin. After sacrificing them, the bone callus was evaluated with axial tomography and classified into three groups: no fusion (0), partial/incomplete fusion (1) and fusion (2). The samples were also histologically analyzed. The associations between the presence of fusion (complete/incomplete) and the group tested were estimated using Poisson log-linear models with two covariates. Adherences to histologically obtained responses were studied using contingency tables and $\chi^{2}$ tests. The significance level was set equal to 0.05 . Results: Treatment with vancomycin has $30 \%(0.30$. CI 95\%: 0.12-0.94) less chance compared to the placebo group, of presenting complete fusion. In other words, the vancomycin group has 2.3 times (CI 95\%: 1.02-4.91) more likelyhood, compared to placebo, to have incomplete fusion. Conclusion: The application of vancomycin powder mixed with graft reduces fusion rates by $30 \%$, but at doses 5 times higher than those routinely used in arthrodesis by the authors' medical team.

Keywords: Fusion, vancomycin, graft, revision.

\section{Nivel de evidencia: I}

* Departamento de Cirugía Espinal. Sanatorio Allende, Córdoba, Argentina.

Correspondencia:

Joint H Guimbard Pérez

Independencia 757, 1er piso,Córdoba, CP 5000, Argentina.

Tel: +543518001153

E-mail: jguimbardmd@hotmail.com

Citar como: Guimbard-Pérez JH, Nicolás-Ortiz P, Cristiani-Winer M, Orosco-Falcone D, Gutiérrez N, Pomba M. Aplicación de vancomicina en polvo sobre el injerto. ¿Afecta la fusión de columna en conejos? Acta Ortop Mex. 2020; 34(5): 276-281. https://dx.doi.org/10.35366/97988 


\section{Introducción}

La infección en las cirugías de columna es una complicación mayor, que afecta negativamente a los pacientes con un impacto profundo en su calidad de vida, conduciendo a un tratamiento antibiótico prolongado, incrementándose el número de reoperaciones, retraso en la rehabilitación e incremento de la morbimortalidad. ${ }^{1,2,3,4}$ Los antibióticos perioperatorios reducen esta tasa de infección, pero en muchas oportunidades la concentración de antibióticos no es la adecuada en los hematomas del sitio quirúrgico. ${ }^{1,2} \mathrm{Con}$ el objetivo de maximizar esa concentración de antibiótico en la herida quirúrgica, desde el año 2011, numerosos estudios han demostrado eficacia y seguridad en niños y adultos ante la administración tópica de vancomicina en el sitio quirúrgico, reduciendo la tasa de infección postquirúrgica en cirugía de columna, con menores efectos adversos que en la administración endovenosa. ${ }^{5,6,7}$

A pesar de lo anterior, el efecto de la vancomicina tópica sobre la osteogénesis continúa siendo controversial, al tratarse de una preocupación respaldada por estudios in vitro, los cuales sugieren que la vancomicina a altas dosis es citotóxica para los osteoblastos. ${ }^{1,2,8,9,10,11,12}$ No obstante, y a partir de diversos estudios clínicos in vivo, no se ha determinado el efecto de la vancomicina en la osteogénesis, ni tampoco se ha aportado una conclusión sobre la tasa de artrodesis espinal. ${ }^{6,13,14}$

Esta información es fundamental para la formulación de protocolos con el fin de prevenir infecciones en el sitio quirúrgico, sin generar efectos adversos en la osteogénesis, logrando artrodesis sólidas. Así, el objetivo del presente trabajo fue explorar el efecto de la vancomicina tópica sobre la fusión espinal en conejos blancos neozelandeses, en un modelo de artrodesis lumbar posterolateral.

\section{Material y métodos}

Se condujo un estudio experimental randomizado doble ciego, el cual fue aprobado y autorizado por el comité de ética institucional en Marzo de 2016. Se realizó cirugía espinal a 30 conejos blancos adultos machos neozelandeses (fusión posterolateral intertransverso unilateral mono segmentario), divididos aleatoriamente en dos grupos, grupo A: únicamente injerto autólogo más placebo, grupo B: injerto autólogo más $200 \mathrm{mg}$ de vancomicina. La edad de los conejos osciló entre 10 a 19 meses, con pesos entre 2.25 a $3.50 \mathrm{~kg}$.

El trabajo fue dividido en tres equipos, con tres integrantes médicos en cada uno, supervisados por un coordinador general y grupal (cirujano espinal de más de dos años de experiencia). Los grupos de profesionales llevaron a cabo las siguientes tareas: rasurado y anestesia; procedimiento quirúrgico propiamente dicho y cierre de heridas y cura plana. Se contó, además, con un observador independiente en la sala de procedimientos, denominado observador $1(\mathrm{O} 1)$.

Previo a la intervención quirúrgica, un farmacéutico realizó el fraccionamiento de 200 mg de vancomicina (15 cáp- sulas) y de $200 \mathrm{mg}$ de lactulosa (placebo 15 cápsulas), fracciones identificadas por un código sólo conocido por el O1.

Antes de la intervención quirúrgica, cada espécimen fue identificado con un precinto, un número y una jaula individual donde el $\mathrm{O} 1$ almacenó la información del número del conejo, peso de los mismos e indicó a cuál le correspondía placebo o vancomicina. Por otro lado, para realizar las evaluaciones participaron dos especialistas en diagnóstico por imagen $(\mathrm{O} 2$ y $\mathrm{O} 3)$ y un especialista en anatomía patológica (O4); ningún integrante del equipo quirúrgico, ni los evaluadores independientes, a excepción de $\mathrm{O} 1$, tuvieron conocimiento de la pertenencia de cada individuo, constituyendo de esa manera un estudio doble ciego.

Los conejos fueron evaluados en el preoperatorio para descartar la presencia de enfermedades o malformaciones. Habitaron en jaulas individuales a una temperatura ambiente de $22-5^{\circ} \mathrm{C}$ y fueron alimentados con comida balanceada específica (150-200 gramos/día y con agua a demanda).

El procedimiento quirúrgico comenzó con la inducción anestésica con ketamina intramuscular $(1 \mathrm{mg} / \mathrm{kg}$ de peso) y diazepam intramuscular (3-5 $\mathrm{mg} / \mathrm{kg}$ peso). La asepsia de la piel se realizó con iodopovidona. Previo a la cirugía los animales permanecieron en ayuno de alimentos y de hidratación por ocho y dos horas respectivamente. Se utilizó autoinjerto óseo obtenido de la cresta ilíaca de cada conejo. En los dos grupos se utilizó cefalomicina $1 \mathrm{mg} / \mathrm{kg}$ intramuscular, una dosis previa a la cirugía y una postquirúrgica. El cirujano y los ayudantes utilizaron guantes estériles en todos los procedimientos, asepsia antisepsia con iodopovidona, decúbito prono, luego abordaje longitudinal posterior, de aproximadamente $5 \mathrm{~cm}$ desde L4-L7 con bisturí tamaño 24, disección prolija por planos llegando a las goteras lumbares bilateralmente, lado derecho o izquierdo a $4 \mathrm{~cm}$ paramediana, hasta identificar goteras y las apófisis transversas unilateralmente monosegmentarias, dejándolas preparadas para la realización del cruentado de la lámina y apófisis transversas, con drill a baja velocidad. Una vez preparado el lecho se procedió a tomar injerto autólogo de una de las crestas ilíacas del conejo, el mismo fue preparado moliéndolo, en chips óseos menores a $0.5 \mathrm{~cm}$. El O1 procedió a abrir la cápsula aleatorizada, volcando el polvo (placebo o vancomicina) sobre el injerto. Se colocó el espécimen a nivel posterolateral e intertransverso unilateral mono segmentario en todos los conejos (Figura 1). Sólo el O1 recogió todas las observaciones. Se efectuó el cierre por planos y cura plana con iodopovidona.

A todos los conejos se les permitió el movimiento adentro de las jaulas, sin restricciones. A las 12 semanas, éstos fueron sacrificados, evaluando las características del callo óseo a nivel intertransverso, mediante tomografía axial computada por los observadores independientes especialistas en imágenes $(\mathrm{O} 2$ y $\mathrm{O} 3)$ donde se les clasificó en tres grupos 0-1-2, en donde 0: No fusión (sin continuidad del puente óseo) 1: Posiblemente fusionado (fusión incompleta o puente irregular) y 2 : fusionado (continuidad del puente 
óseo). Un especialista en anatomía patológica (O4) evaluó las muestras histológicamente, los cortes de los especímenes se obtuvieron seccionando en el punto medio del aloinjerto, y que fueron incluidos en parafina. Múltiples cortes de aproximadamente $6 \mathrm{~mm}$ de espesor fueron realizados con micrótomo y teñidos con hematoxilina eosina para microscopía de luz convencional. Se evaluaron como respuestas la osteointegración, formación de callo óseo, reabsorción del injerto, vascularización/angiogénesis, fibrosis y la presencia de exudado fibrinoleucocitario.

Se realizó un análisis descriptivo global (tablas de frecuencia y gráficos específicos) de cada característica (presencia de fusión completa y de fusión incompleta o parcial) y para cada grupo (placebo y vancomicina) y luego diferenciando entre observadores. Se estimaron medidas de asociación para dichas variables categóricas (coeficientes de similaridad). Las asociaciones entre la presencia de fusión (completa o parcial) y las variables recolectadas y grupo ensayado (placebo como línea de base) fueron analizadas mediante modelos log-lineales Poisson con dos covariables.

La adherencia con las respuestas obtenidas por el observador a nivel histológico fue estudiada usando tablas de contingencia y test de $\chi^{2}$ (Fisher cuando las celdas de frecuencias estimadas contenían valores inferiores a 5). Se utilizó un nivel de significación igual a 0.05 . Los análisis fueron llevados a cabo en STATA v. 14 (Stata Corp, Texas, 2014).

\section{Resultados}

Luego del procedimiento, en el transcurso de los primeros 10 días murieron tres especímenes, por lo tanto, de los 30 conejos se analizaron 27, de esos especímenes 14 fueron del grupo A: placebo y 13 fueron del grupo B: vancomicina. A las 12 semanas se sacrificaron los animales y a continuación se exponen los principales resultados obtenidos de los análisis llevados a cabo con el fin de investigar el efecto de la vancomicina tópica sobre la fusión espinal en dos conjuntos de conejos blancos neozelandeses y a partir de un modelo de artrodesis posterolateral.
Primero se describen las características generales sobre la fusión (Figura 2), considerando dos análisis independientes (dos observadores) y luego la valoración de la osteointegración evaluando histológicamente las muestras. Se señala que luego de la aplicación de ambos tratamientos, tres especímenes murieron $(10 \%$ de la muestra total).

El 80 y $60 \%$ de las muestras, para los tratamientos A y B, respectivamente, presentó fusión completa, existiendo una leve tendencia a detectar diferencias significativas en esas proporciones $(\mathrm{p}=0.079)$, siendo que 13 y $27 \%(\mathrm{p}=0.361)$, correspondieron a la valoración parcial, apenas.

Este patrón fue diferente cuando la respuesta provino del segundo observador (53 y 67\% de fusión completa para A y $\mathrm{B}$ respectivamente, $\mathrm{p}=0.456 ; 40 \%$ y $20 \%$ de fusión parcial, $\mathrm{p}=0.197)$. Las Figuras 3 y 4 ilustran el patrón diferencial de las frecuencias observadas para la valoración de la fusión, para cada observador y en cada tratamiento. Se puede observar que las grandes discordancias $(p=0.003)$ entre los observadores se presentan en la valoración de la fusión (completa o incompleta/parcial) para el tratamiento injerto autólogo con placebo.

Al comparar con la valoración de la osteointegración, a nivel histológico, las proporciones clasificadas como completas no difirieron entre los tratamientos $(0.76$ y 0.85 para A y B, respectivamente, $\mathrm{p}=0.557$, Figura 5) y no tienen concordancia con la respuesta proveniente de los observadores $(\mathrm{p}=0.023)$. Esta falta de adherencia con el estándar de oro es básicamente para las valoraciones en el tratamiento con vancomicina $(\mathrm{p}=0.021)$.

Así, considerando como más específica la respuesta otorgada por el primer observador, el tratamiento con vancomicina tiene 30\% (IC 95\%: 0.12-0.94) menos probabilidad, respecto del tratamiento que sólo recibió placebo, de presentar fusión completa. En otros términos, el grupo que recibió injerto autólogo con vancomicina tiene 2.3 veces (IC 95\%: 1.02-4.91) más de oportunidad, respecto del placebo, de ser clasificado con fusión incompleta o parcial.
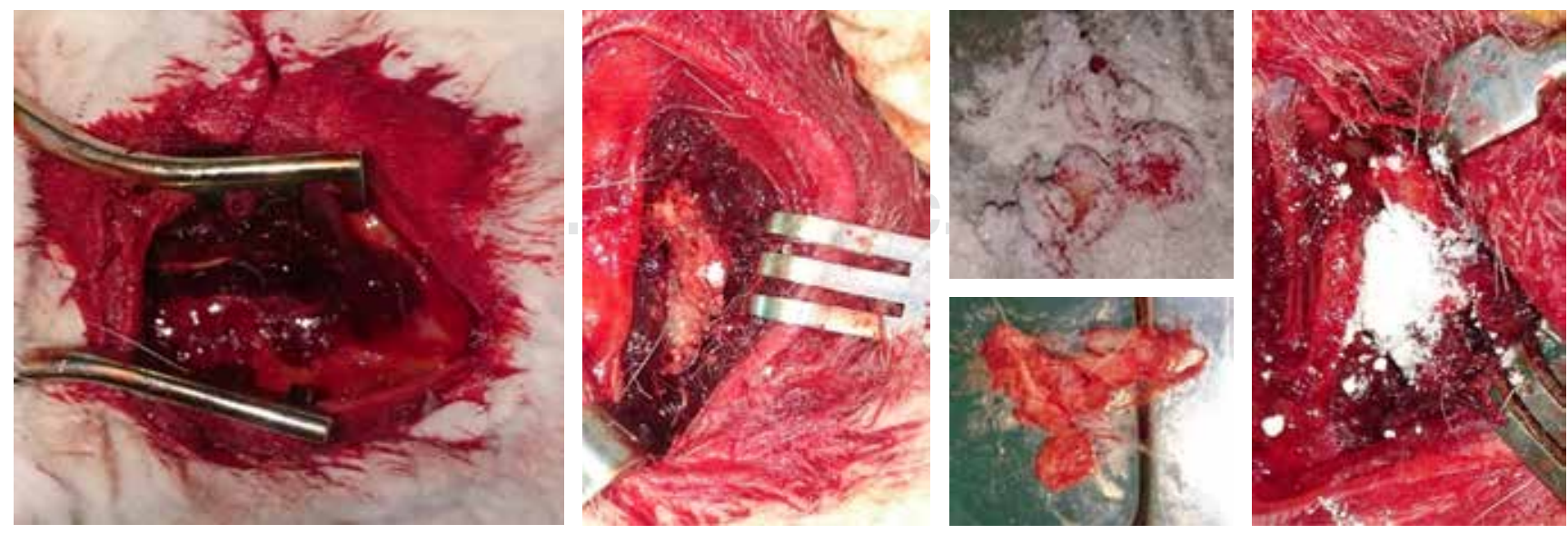

Figura 1: Imágenes operatorias del lecho quirúrgico tras la aplicación de vancomicina en el injerto y lecho quirúrgico. 

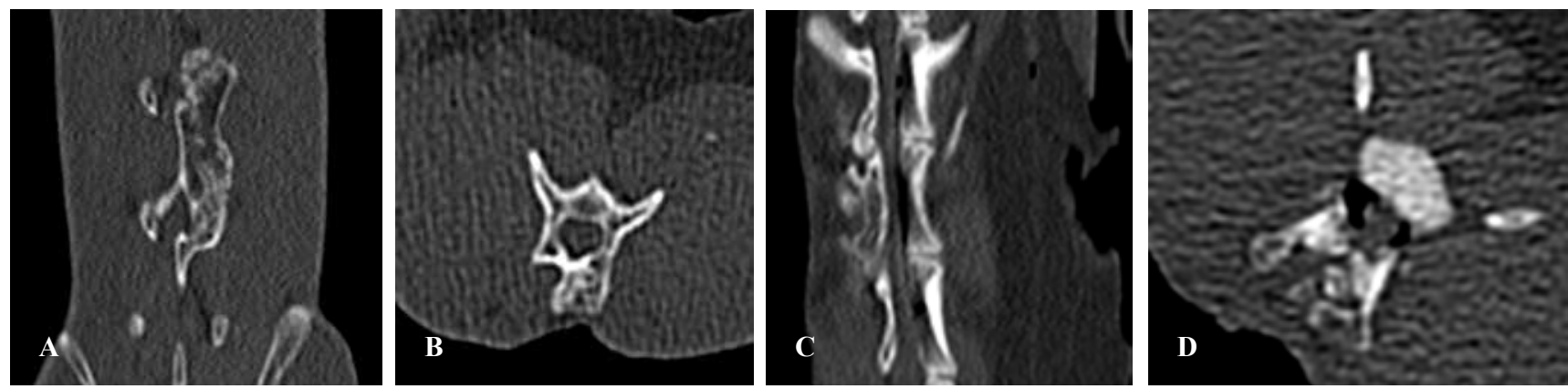

Figura 2: Tomografía axial computada. A y B) Grupo de injerto autólogo más placebo (fusión completa). C y D) Grupo de injerto autólogo más vancomicina (fusión parcial).

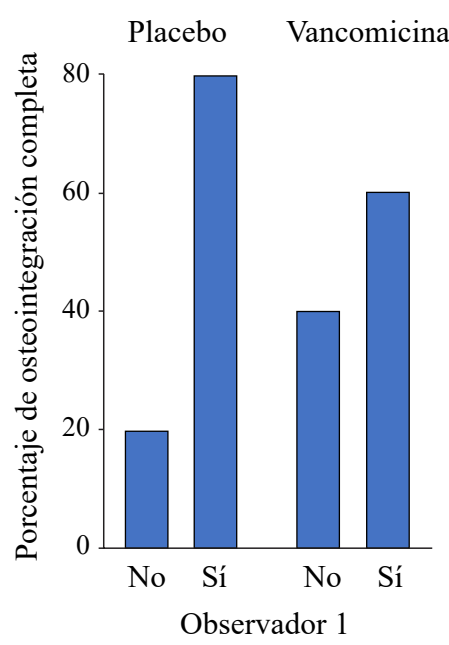

Figura 3: Distribución de frecuencias de la respuesta fusión completa en los tratamientos de injerto autólogo con placebo y con vancomicina, según observador.

\section{Discusión}

A pesar del uso de la terapia sistémica con antibióticos y las técnicas de esterilidad, las infecciones del sitio quirúrgico siguen siendo una gran preocupación para los cirujanos y pueden tener un efecto devastador en los resultados. Numerosos estudios han demostrado que la vancomicina en polvo mezclada con el injerto reduce significativamente la incidencia de infección postoperatoria, pero hay escasa bibliografía y cierta controversia en el efecto que presenta sobre la osteogénesis. ${ }^{6,15}$

La migración celular es un evento biológico clave en la formación y remodelación de tejidos. Por lo tanto, se requiere un suministro continuo de osteoblastos para la remodelación ósea y la consolidación de fracturas. Todas las dosis de vancomicina ensayadas in vitro en el trabajo de Eder y col. inhibieron significativamente el potencial de migración de los osteoblastos y, por lo tanto, podrían interferir con la remodelación ósea in vivo. La inhibición de la migración celular puede explicarse por una supresión en la formación de seudópodos, que es el mecanismo más común de locomoción de células eucarióticas. ${ }^{1,13}$
Hay otros estudios in vitro que sugieren que la vancomicina en altas dosis es tóxica para diferenciar los osteoblastos, sin embargo, en el estudio de Edin y col. sugieren que solamente más de $10 \mathrm{mg}$ de vancomicina ya causa muerte celular. ${ }^{1,2,8,9,10,11,16,17,18}$

La aplicación de vancomicina en dosis medias y altas dio como resultado una disminución significativa del $\mathrm{pH}$ y, por el contrario, para la remodelación ósea in vivo se requieren niveles alcalinos de $\mathrm{pH}$, por lo que podría ser otro factor que disminuya la osteogénesis. ${ }^{19}$

Estudios actuales, clínicos, no han logrado determinar definitivamente el efecto de la vancomicina en la tasa de seudoartrosis. ${ }^{6,14,15}$ Sin embargo, en el metaanálisis de Alcala-Cerra y col. solamente tres estudios analizan el riesgo de seudoartrosis luego de haber utilizado vancomicina en polvo; incluyeron entre los tres trabajos un total de 2,156 pacientes, donde a pesar de las teorías que señalan el potencial efecto tóxico que podrían tener las altas concentraciones de vancomicina dentro de la herida quirúrgica sobre los osteoblastos, el análisis del RR (riesgo relativo) combinado indica que no existe diferencia estadísticamente significativa en el riesgo de seudoartrosis, lo cual posiblemente esté

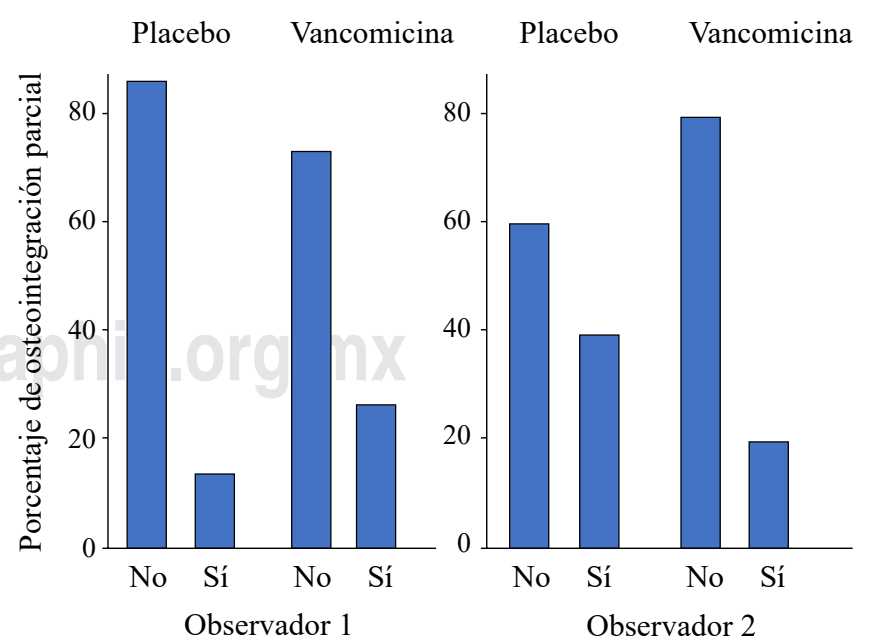

Figura 4: Distribución de frecuencias de la respuesta fusión parcial en los tratamientos de injerto autólogo con placebo y con vancomicina, según observador. 


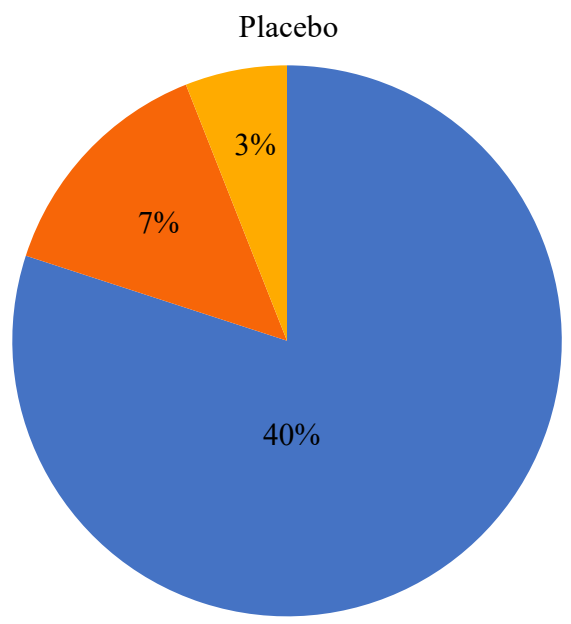

Integración según histología

Completa

Parcial

relacionado con la rápida repoblación de estas células en el lecho de fusión, tan pronto las concentraciones de vancomicina disminuyen rápidamente dentro de la herida quirúrgica. Las limitaciones de este metaanálisis es que fue observacional, siendo lo ideal estudios controlados aleatorizados, y además fue un bajo número de estudios (3) que evaluaron fusión/seudoartrosis. ${ }^{18}$

A su vez, en el trabajo de Mendoza y col. en un modelo in vivo, en roedores, con un defecto femoral, se incluyó un grupo de alta dosis de vancomicina, para evaluar los posibles efectos de concentraciones excesivas, pero no se encontraron diferencias significativas en los puntajes de fusión, sin embargo, es importante tener presente las diferencias anatómicas entre las columnas de rata y humanas y que la curación ósea en ratas generalmente ocurre a un ritmo más rápido y por lo tanto menos susceptible a factores inhibidores. ${ }^{11}$ En consecuencia, debido a los distintos patrones de curación, la vancomicina podría tener un efecto diferente en los humanos y pueden no tener un correlato directo en el escenario clínico.

Una publicación actual de Zhang y col. en tibias de conejos neozelandeses utilizando altas dosis de vancomicina, similares al presente trabajo, observan una disminución significativa de la masa ósea y de la secreción de osteocalcina. ${ }^{20}$

En esta investigación, con el objetivo de aclarar el efecto de la vancomicina en la fusión, se utilizaron conejos con una dosis de vancomicina alta ( 5 veces mayor que la utilizada rutinariamente en los procedimientos de fusión), en donde los resultados obtenidos sugieren fuertemente que la aplicación local de vancomicina influye negativamente en la curación ósea y fusión espinal. Algunas limitaciones que resultan del presente trabajo fueron: el número de especímenes incluidos y ajustar la dosis de vancomicina para lograr conocer la concentración mínima requerida para evitar infecciones y para conocer el umbral crítico por encima del cual la vancomicina inhibe la curación ósea.
Vancomicina

Figura 5:

Distribución de frecuencias de la clasificación de la fusión en los tratamientos de injerto autólogo con placebo y con vancomicina.

\section{Conclusión}

La aplicación de vancomicina en polvo, mezclada con injerto autólogo, reduce la formación ósea y las tasas de fusión $30 \%$ pero a dosis cinco veces más alta que las usadas rutinariamente en los procedimientos de artrodesis o fusión por el equipo médico de los autores. Por lo tanto, se considera que es tóxico para los osteoblastos y es fundamental para la formulación de protocolos para prevenir infecciones en el sitio quirúrgico sin generar efectos adversos en la osteogénesis.

\section{Referencias}

1. Eder C, Schenk S, Trifinopoulos J, Külekci B, Kienzl M, Schildböck $\mathrm{S}$, et al. Does intrawound application of vancomycin influence bone healing in spinal surgery? Eur Spine J. 2016; 25(4): 1021-8.

2. Philp AM, Raja S, Philp A, Newton Ede MP, Jones SW. The effect of vancomycin and gentamicin antibiotics on human osteoblast proliferation, metabolic function, and bone mineralization. Spine (Phila Pa 1976). 2017; 42(3): 202-7.

3. Savage JW, Anderson PA. An update on modifiable factors to reduce the risk of surgical site infections. Spine J. 2013; 13(9): 1017-29.

4. Godil SS, Parker SL, O'Neill KR, Devin CJ, McGirt MJ. Comparative effectiveness and cost-benefit analysis of local application of vancomycin powder in posterior spinal fusion for spine trauma: clinical article. J Neurosurg Spine. 2013; 19(3): 331-5.

Armaghani SJ, Menge TJ, Lovejoy SA, Mencio GA, Martus JE. Safety of topical vancomycin for pediatric spinal deformity: nontoxic serum levels with supratherapeutic drain levels. Spine (Phila Pa 1976). 2014; 39(20): 1683-7.

6. Chiang HY, Herwaldt LA, Blevins AE, Cho E, Schweizer ML. Effectiveness of local vancomycin powder to decrease surgical site infections: a meta-analysis. Spine J. 2014; 14(3): 397-407.

7. O'Neill KR, Smith JG, Abtahi AM, Archer KR, Spengler DM, McGirt $\mathrm{MJ}$, et al. Reduced surgical site infections in patients undergoing posterior spinal stabilization of traumatic injuries using vancomycin powder. Spine J. 2011; 11(7): 641-6.

8. Molinari RW, Khera OA, Molinari WJ 3rd. Prophylactic intraoperative powdered vancomycin and postoperative deep spinal wound infection: 
1,512 consecutive surgical cases over a 6-year period. Eur Spine J. 2012; 21 Suppl 4(Suppl 4): S476-82.

9. Caroom C, Tullar JM, Benton EG Jr, Jones JR, Chaput CD. Intrawound vancomycin powder reduces surgical site infections in posterior cervical fusion. Spine (Phila Pa 1976). 2013; 38(14): 1183-7.

10. Rathbone CR, Cross JD, Brown KV, Murray CK, Wenke JC. Effect of various concentrations of antibiotics on osteogenic cell viability and activity. J Orthop Res. 2011; 29(7): 1070-4.

11. Mendoza MC, Sonn KA, Kannan AS, Bellary SS, Mitchell SM, Singh $\mathrm{G}$, et al. The effect of vancomycin powder on bone healing in a rat spinal rhBMP-2 model. J Neurosurg Spine. 2016; 25(2): 147-53.

12. Newton Ede MP, Philp AM, Philp A, Richardson SM, Mohammad S, Jones SW. Povidone-iodine has a profound effect on in vitro osteoblast proliferation and metabolic function and inhibits their ability to mineralize and form bone. Spine (Phila Pa 1976). 2016; 41(9): 729-34.

13. Goldschmidt E, Rasmussen J, Chabot JD, Gandhoke G, Luzzi E, Merlotti L, et al. The effect of vancomycin powder on human dural fibroblast culture and its implications for dural repair during spine surgery. J Neurosurg Spine. 2016; 25(5): 665-70.

14. Sweet FA, Roh M, Sliva C. Intrawound application of vancomycin for prophylaxis in instrumented thoracolumbar fusions: efficacy, drug levels, and patient outcomes. Spine (Phila Pa 1976). 2011; 36(24): 2084-8.
15. Kang DG, Holekamp TF, Wagner SC, Lehman RA Jr. Intrasite vancomycin powder for the prevention of surgical site infection in spine surgery: a systematic literature review. Spine J. 2015; 15(4): 762-70.

16. Edin ML, Miclau T, Lester GE, Lindsey RW, Dahners LE. Effect of cefazolin and vancomycin on osteoblasts in vitro. Clin Orthop Relat Res. 1996; (333): 245-51.

17. Ketonis C, Barr S, Adams CS, Shapiro IM, Parvizi J, Hickok NJ. Vancomycin bonded to bone grafts prevents bacterial colonization. Antimicrob Agents Chemother. 2011; 55(2): 487-94.

18. Alcalá-Cerra G, Paternina-Caicedo AJ, Moscote-Salazar LR, Gutiérrez-Paternina JJ, Niño-Hernández LM. Application of vancomycin powder into the wound during spine surgery: systematic review and meta-analysis. Rev Esp Cir Ortop Traumatol. 2014; 58(3): 182-91.

19. Lee GH, Hwang JD, Choi JY, Park HJ, Cho JY, Kim KW, et al. An acidic $\mathrm{pH}$ environment increases cell death and pro-inflammatory cytokine release in osteoblasts: the involvement of BAX inhibitor-1. Int J Biochem Cell Biol. 2011; 43(9): 1305-17.

20. Zhang Y, Shen L, Mao Z, Wang N, Wang X, Huang X, et al. Icariin enhances bone repair in rabbits with bone infection during post-infection treatment and prevents inhibition of osteoblasts by vancomycin. Front Pharmacol. 2017; 8: 784. 